\title{
Geographies of Information Inequality in Sub-Saharan
}

\section{Africa}

Mark Graham and Christopher Foster

Oxford Internet Institute, University of Oxford

Network Inclusion Roundtable - Bangalore, India - October 2014

\section{1) Introduction: Promises of Changing Connectivity}

Sub-Saharan Africa has traditionally been characterised by stark barriers to telecommunication and flows of information. Rates for long distance phone calls throughout Sub-Saharan Africa (SSA) used to be some of the highest in the world, and Internet costs and speeds similarly were out of the reach of all but the most privileged citizens. However, in the last few years, there have been radical changes to SSA's international connectivity. Fibre-optic cables have been laid throughout the continent and there are now over one hundred and fifty million Internet users and over seven hundred million mobile users in the region.

This rapid transformation in the region's connectivity has encouraged politicians, journalists, academics, and citizens to speak of an ICT-fuelled revolution happening on the continent. Individuals and firms would increasingly be linked into global networks - interacting, selling and using knowledge through this connectivity (Graham \& Mann 2013).

This has also been reflected in new ambitions and policy in SSA. For example, in Rwanda (a strong advocate of upgrading connectivity to drive development) the stated policy goal has been to:

"transform her subsistence agriculture dominated economy into a service-sector driven high value-added information and knowledge economy that can compete on the global market" (GoR 2001 p.7)

Changing connectivity thus is articulated as a core driver of wider economic change in SSA. It is seen as providing a path for the region to move away from reliance on agriculture and extractive industries and towards a focus on the quaternary and quinary sectors (in other words, the knowledge-based parts of the economy).

However, while much research has been conducted into the impacts of ICTs on older economic processes and practices, there remains surprisingly little research into the emergence of the new informationalised economy in Africa. As such, it is precisely now that we urgently need research to understand what impacts are observable, who benefits, who doesn't, and how these changes match up to our expectations for change. We need to ask if 
we are seeing a new era of development on the continent fuelled by ICTs, or whether SubSaharan Africa's engagement with the global knowledge economy continues to be on terms that reinforce dependence, inequality, underdevelopment, and economic extraversion.

We begin to address this issue by synthesising the outputs of two multi-year research projects that we have carried out which provide in-depth analysis of SSA connectivity use. The first one addresses the effects of changing connectivities on global geographies of voice, representation, and participation, particularly through exploring the dynamics online platforms, tools and databases. The second project is grounded in in-depth qualitative research, examining the effects of changing connectivity on firms in core sectors of the economy (tea, tourism, and Business Process Outsourcing) in Kenya and Rwanda.

\section{2) Barriers and exclusions}

The suggestion that changing connectivity as a result of the laying of fibre-optic cables would lead to an improvement in internet availability and a reduction in costs in SSA is well supported. Changes in physical connectivity have, in part, led to upwards growth in subscription numbers, as highlighted in Figure 1. However, the degree of catch-up in SSA appears limited, Internet use is still limited and well behind the rest of the world[1].

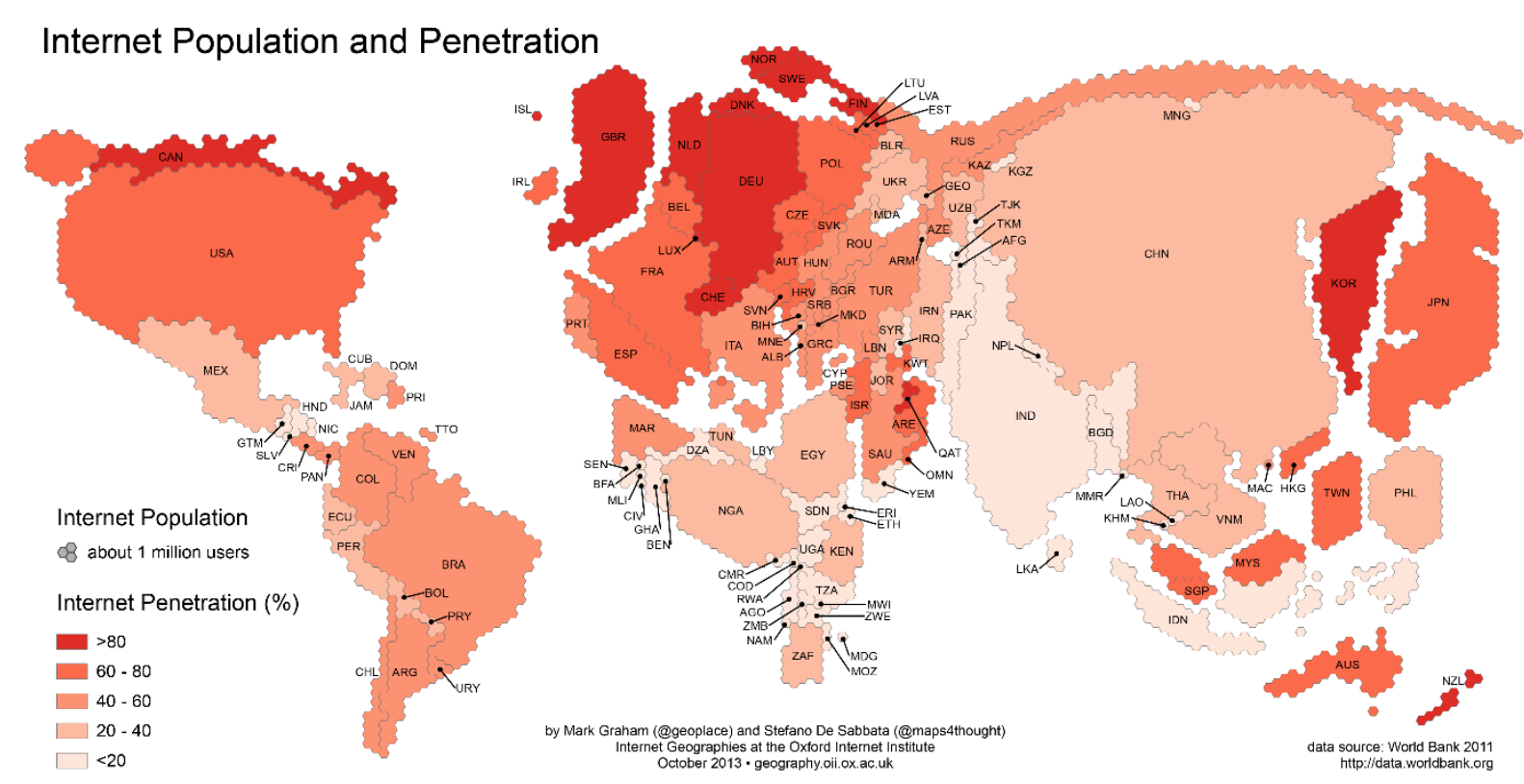

Figure 1: internet population and penetration

Source: Graham \& Sabatta(2013) drawing on World Bank data

This jump in internet access and use, however, does not appear to have been as impactful as many had hoped. In our qualitative work in SSA, existing sectors such as exportorientated commodities and tourism, growing internet use has not fundamentally transformed relationships of production and the distribution of value. Meanwhile, new 
knowledge sectors articulated as the cornerstone of economic 'leap-frogging' appear to comprise of a small number of often struggling firms. Similar outcomes were found in examinations of the geography of coverage and contributions to online platforms. Research on presence of SSA locations within Google searches and Twitter contributions represented minuscule proportions, even allowing for the low penetration.

Our work, in significantly different domains, highlighted barriers that limited the effectiveness of connectivity reducing information inequality. We outline five core categories that conceptualise these barriers: Representation, Contribution, Access-toinformation, Connectivity and Non-neutral networks/technologies. Each of these is detailed below:

\section{2a) Representations}

We found not only stark inequalities in the amount of information produced from and about different parts of the world, but we also discovered indications that older patterns of informational inclusion and exclusion were being reinforced. The issue is not just that much of SSA left out of the representation on online platforms, tools, and databases. It is also that internet users from SSA often focus their attention on the global informational cores (North America and Western Europe): resulting in patterns of increasing informational poverty and richness (Graham et. al. 2014). 

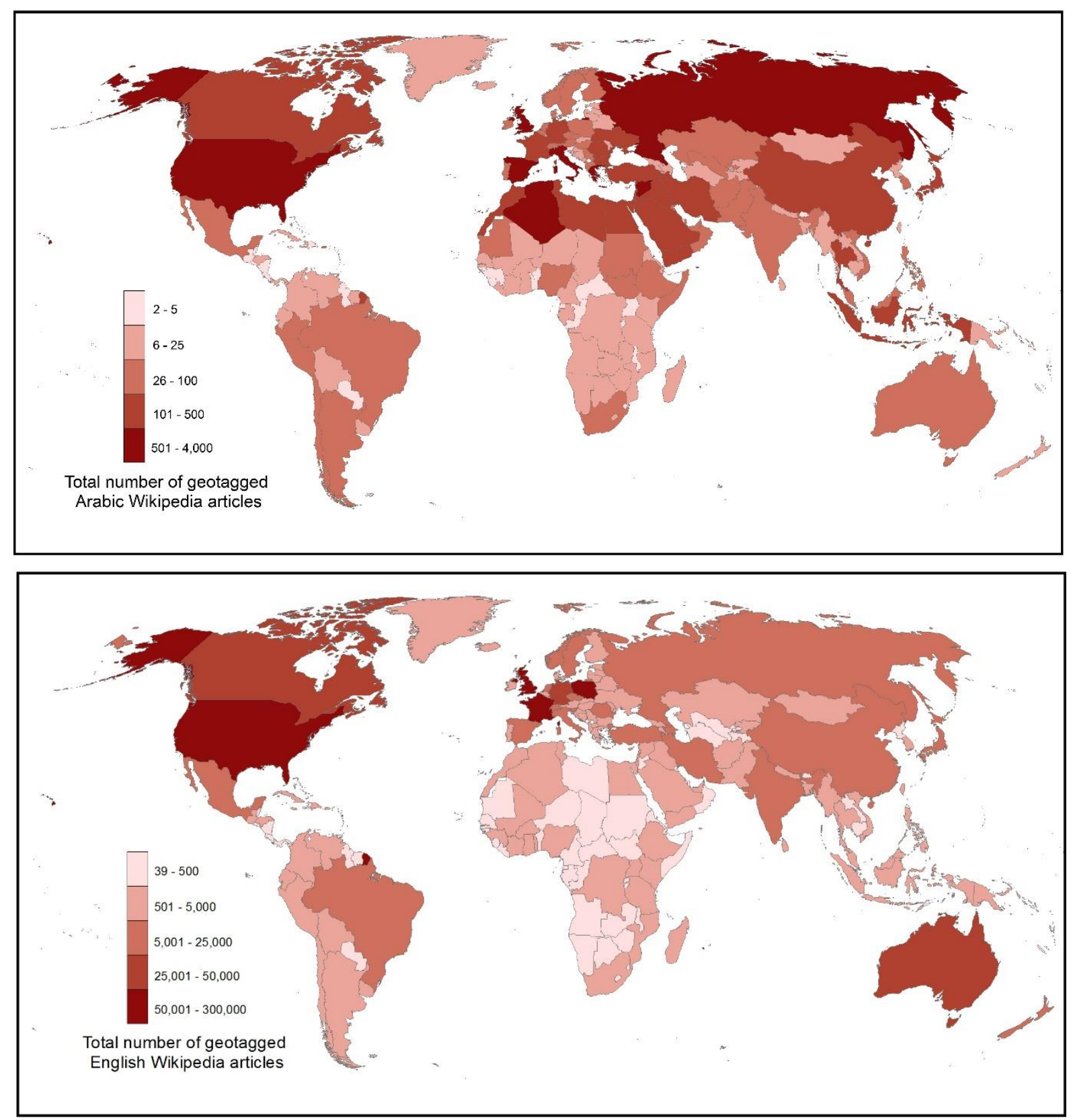

Figure 2: Maps of Articles in Arabic(top) and English(bottom) Wikipedia

Source: Graham et. al. (2014). Note there are more Arabic articles about many European countries than many predominantly Arabic-speaking countries.

Work in the Rwandan tea sector also revealed uneven online representation of processes and products at a more local level. At the level of tea processing and logistics, extensive automation and connection has rendered many parameters of tea production (weight, grade, location of batches) visible online and thus compliant to be analysed, compared and managed. Yet, this was less true for farmer activities. Rural actors involved in tea production are often left relying on guesswork, norms or downward edicts to orientate their growing activities. 
At the broadest level, uneven representation is without a doubt one outcome of the uneven global distribution digital connectivity. At the same time it can also be brought into being by subtle balances of power and choices as highlighted in the tea sector. Representation of knowledge can determine the extent to which information can be employed, applied, put into practice, and integrated into processes. People, places, and practices absent from representations not just lose voice, but also potentially become invisible.

\section{2b) Contributions}

We explored a range of platforms and information repositories and found that only a relatively tiny amount of content hosted in online repositories comes from Sub-Saharan Africa (many of our results and maps can be found at geography.oii.ox.ac.uk). Much of this imbalance can't simply be explained away by uneven levels of online access. For instance, if looking at the registration of domain names, there is a domain for every two internet users; whereas the average in the Middle East and Africa is one domain for every fifty internet users (see map below). Similar patterns are evident on every platform that we looked at (e.g. on Wikipedia there are more contributions that come from Hong Kong than all of Africa combined). Furthermore, this small amount of participation from Sub-Saharan Africa has meant that much of the content created about Sub-Saharan Africa comes from the global informational cores.

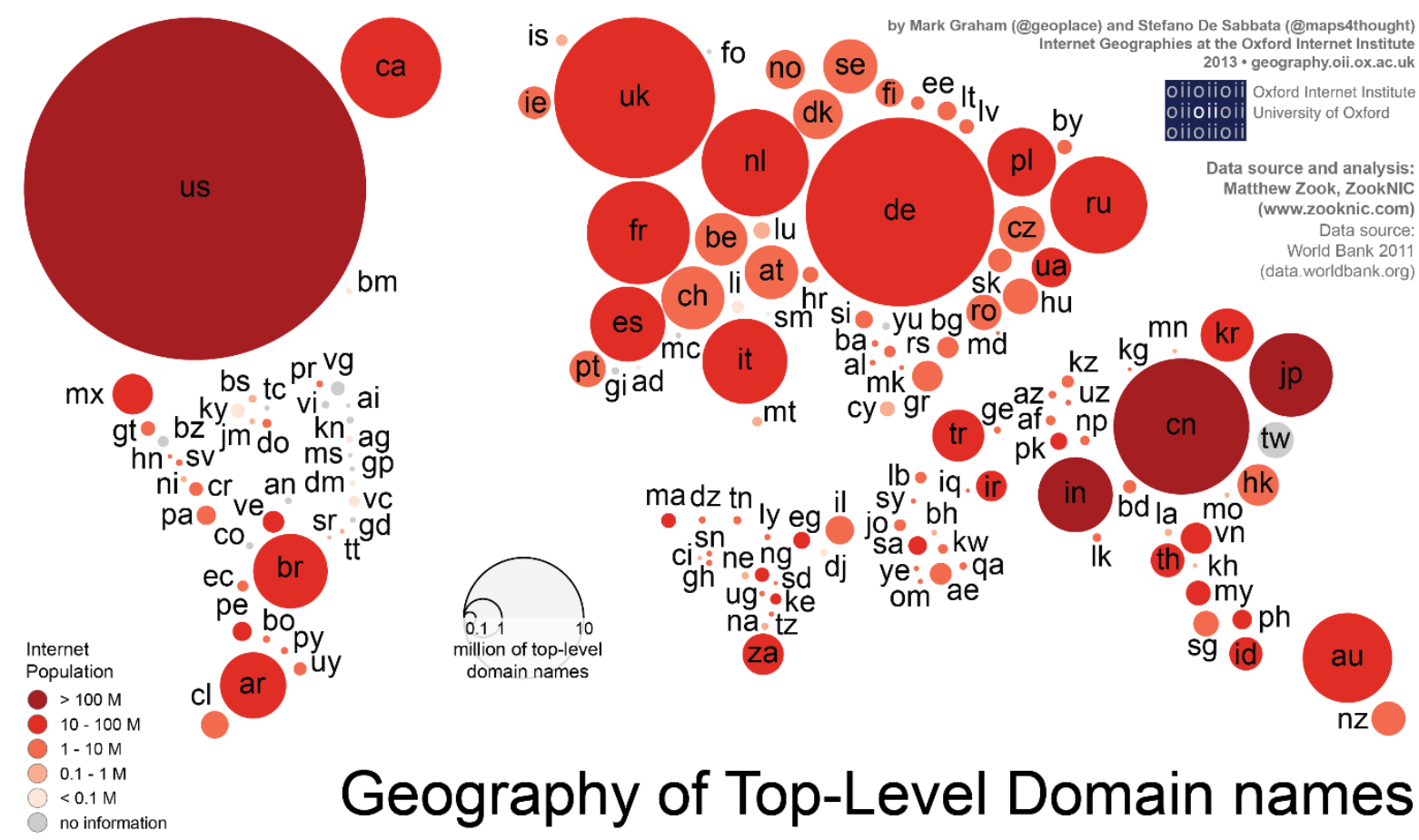

Figure 3: Geography of top level domain names

Source: Graham \& Sabatta(2013) drawing on World Bank data 


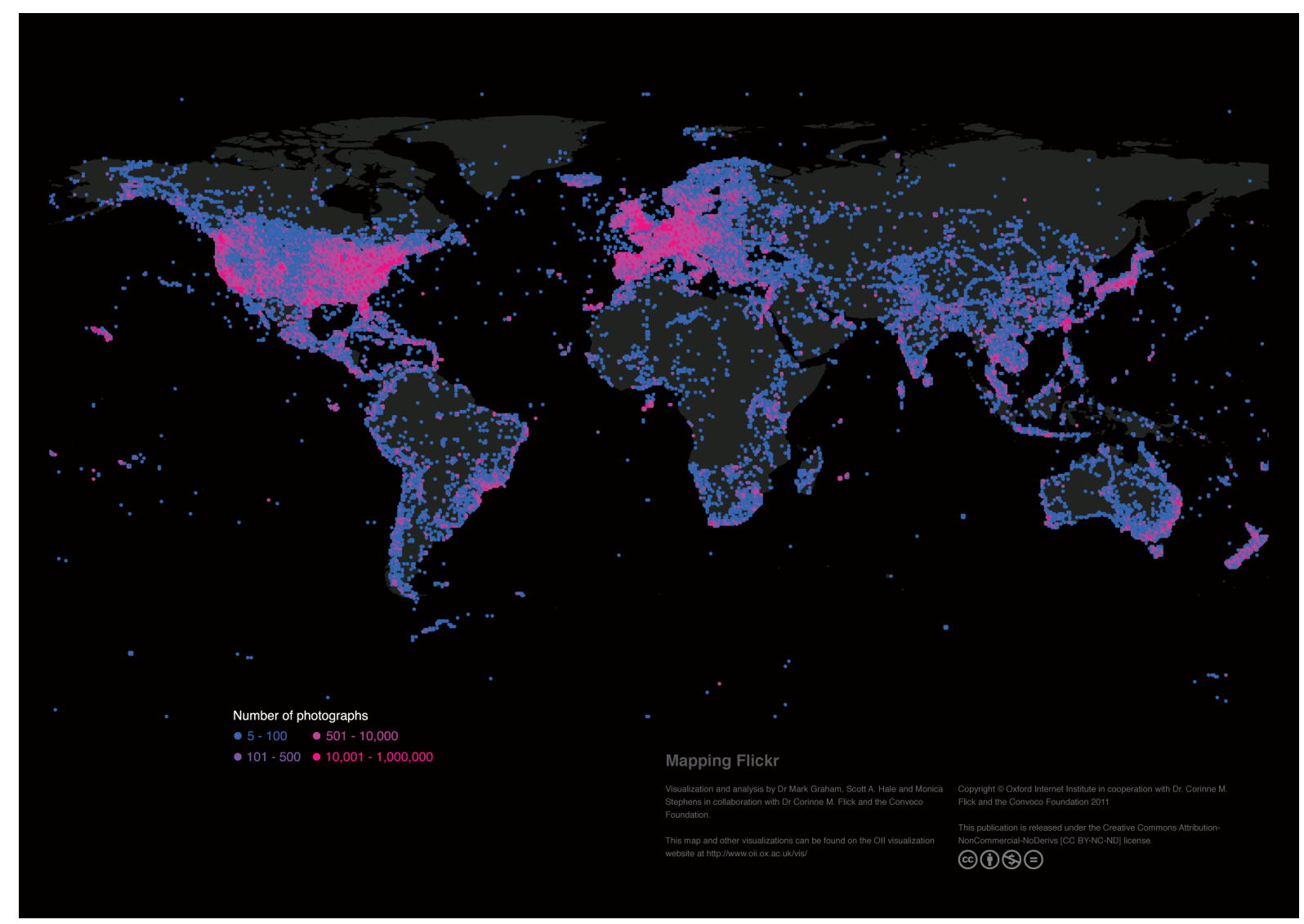

Figure 4: Mapping geo-tagged flickr contributions

Source: Graham, Hale \& Stephens (2013) drawing on flickr data

These findings were also mirrored in research on use of the Internet in agriculture in East Africa related to farming processes and information. Whilst some farmers and intermediary representatives (i.e. Co-operatives, NGOs) were aware of the Internet as a source of new information, a lack of locally created knowledge often skewed the types of advice and solutions available online. Information about planting and quality control available online often came from generic, global resources with less relevance to the specificities and context of the region.

These findings not only point to a lack of representation, but also authorship, voice, contributions, and participation; and highlight the need to pay attention to a more subtle politics of knowledge.

\section{2c) Access to information}

Being able to access ICTs does not imply that relevant information is suddenly available. In the economic sectors that we looked at, we found cases where certain groups were intentionally excluded from information. More often though, lack of access related to issues where suitable information was difficult to access or inappropriate to the context. For example, in the East African tourism sector, Rwandan tour firms are increasingly connected online, but they have been slow in directly linking to tourists. For tour firms, one limit is lack 
of access to information on customers and activities to allow them to build compelling products. Sometimes information is hidden behind paywalls not accessible to these firms but there were also cases where useful information (e.g sources of tourist to the region) were hidden within complex statistics and difficult to understand.

\section{2d) Connectivity}

Even though much infrastructure to support digital connectivity has been built, the ability to digitally connect has distinct geographies. This is especially true if you compare average broadband prices with average yearly income (as we do in the figure below). Doing so reveals stark remaining barriers to connectivity that are particularly experienced in SubSaharan Africa.
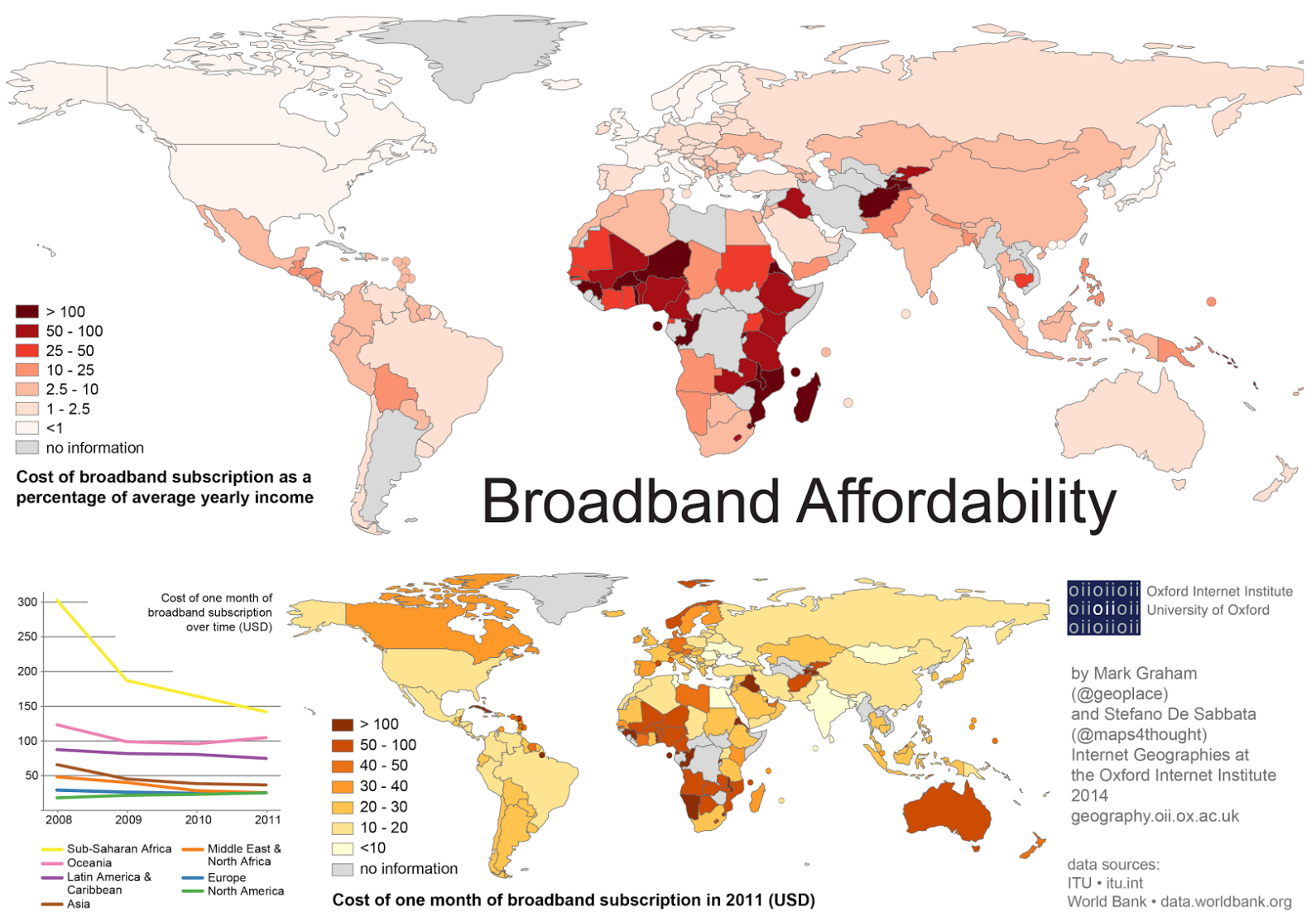

Figure 5: Broadband Affordability

Source: Graham \& Sabatta(2013) drawing on ITU/World Bank data

In East Africa, for instance, even as fibre-optic connections allowed better backbone connections for the region, there was a vast difference between those firms able to link directly to fibre or resilient broadband options, and those who survive using mobile internet access. The latter experienced inconsistent and often saturated connections. These were prone to dropouts and problems related to mobile coverage, service provider issues and liable to be affected by weather conditions. 
This was particularly problematic for smaller firms and entrepreneurs who are looking to interact more directly with customers internationally. Network dropouts and saturation can be detrimental to clear consumer interactions and trust. As some of the barriers to accessing backbone connectivity barriers are reduced, what we are therefore seeing is a move from global to new local inequalities around digital access.

\section{2e) Non-neutral networks/technologies}

Barriers to effective use of infromation also exist in the way that ICTs or networks are 'nonneutral'. That is, they may privilege generation or use of certain forms of information over others. For instance, the lower user contributions to Wikipedia from SSA might be linked to the very governance of Wikipedia: which allows only certain kinds of sources and neglects others (e.g. oral sources).

In similar way, digital systems often work within structured templates (e.g. the design of systems and databases) in order for data to be standardised and relatable. Such templates often poorly fitting to the needs of users in SSA. One instance of this is in the Rwanda tourism sector. Rwandan firms were frequently unable to effectively digitally link up with global travel agents because of their inability to digitally codify their complex activities.

\section{3) Towards deeper interrogation of power}

In sum, changing connectivity has not fully unleashed the effects that many expected it to. These outcomes will come as no surprise for those familiar with some of the critical literature on the 'digital divide' (Norris 2001, Selwyn 2004, Warschauer 2003, Graham 2011). As Warschauer outlined over a decade ago, for digital inclusion, it is necessary to centralise the wider contexts of development,

"an overemphasis on the mere presence of computers or Internet connections, without a corresponding emphasis on social mobilization and transformation, can squander resources while leaving inequity intact processes that underlie social development and inclusion" (Warschauer 2003. p.303)

These critiques form the foundation of our understanding of inclusion in network societies. We need to think about connectivity as not just a technical challenge (i.e. concerns about 'penetration'), but a socio-technical one (i.e. skills, costs, culture, etc.).

Our work highlights a further step needed, that goes beyond these rather functional and instrumental approaches to connectivity, towards more dynamic perspectives on power within the network society (Prey 2012). Put differently, the focus should not necessarily be on simple measure of inclusion or exclusion - but rather on power in networks. Our very understanding of 'connectivity' could therefore be broadened to incorporate both the ways 
that links/connections/connectivities consolidate or distribute power, and the ways that that power in manifested in voice, representation, and the capture and creation of value.

From such a perspective, many of the barriers in online representation, contribution, access and connectivity that we have observed appear to represent a shift in power away from Sub-Saharan African firms and individuals.

\section{4) Conclusion}

Ultimately, this work is a beginning to think about what connectivity means to inclusion in the 'network society.' Connectivity certainly isn't a sufficient condition for inclusion and equity, and we need to ask whether it is a necessary one.

Connectivity, rather, tends to be an amplifier: one that often reinforces rather than reduces inequality. We therefore need to move towards deeper critical socio-economic interrogations of the barriers or structures that limit activity and reproduce digital inequality. The categorisations developed here offer an empirically-driven and systematic way to understand these barriers in more detail.

As the next generation of connectivity projects (e.g. 4G, Facebook's drones, Google's balloons etc.) again draw on the same skewed expectations and goals, we need to loudly call for a more critical approach to understanding such projects. Rhetoric about connectivity needs to be accompanied by a wider debate about how changing connectivities might actually facilitate change in the world, and who changing connectivities might ultimately benefit.

\section{Acknowledgements}

This work was also made possible by research grants from the International Development Research Centre, the ESRC/DFID ( Grant reference (RES-167-25-0701) and ESRC reference $(E S / 1033777 / 1))$, and the ERC.

\section{References}

GoR (2001) National ICT Strategy and Plan NICl, Government of Rwanda, Kigali, Rwanda.

Graham, M., Hogan, B., Straumann, R. K., and Medhat, A. 2014. Uneven Geographies of UserGenerated Information: Patterns of Increasing Informational Poverty. Annals of the Association of American Geographers (forthcoming). 
Graham, M. \& Mann, L. (2013) Imagining a Silicon Savannah? Technological and Conceptual Connectivity in Kenya's BPO and Software Development Sectors. The Electronic Journal of Information Systems in Developing Countries, 56.

Graham, M. 2011. Time Machines and Virtual Portals: The Spatialities of the Digital Divide. Progress in Development Studies. 11 (3). 211-227.

Norris, P. (2001) Digital Divide: Civic Engagement, Information Poverty, and the Internet Worldwide. Cambridge University Press.

Prey, R. (2012) The Network's Blindspot: Exclusion, Exploitation and Marx's ProcessRelational Ontology. TripleC (Cognition, Communication, Co-Operation): Open Access Journal for a Global Sustainable Information Society, 10(2).

Selwyn, N. (2004) Reconsidering Political and Popular Understandings of the Digital Divide. New Media \& Society, 6(3), p. 341.

Warschauer, M. (2003) Dissecting the“ Digital Divide": A Case Study in Egypt. The Information Society, 19(4), pp. 297-304. 